\title{
D006
}

\section{A Novel Approach to Incorporate Full Petroleum System Analysis into Play Risk Assessments}

\section{Bryant* (Schlumberger), T. Levy (Schlumberger), M. Neumaier (Schlumberger) \& N. Tessen (Schlumberger)}

\section{SUMMARY}

In this paper we illustrate a new methodology to derive play chance maps for all elements of the petroleum system - by converting maps of physical properties and interpretations to play chance maps, which are then combined to give an assessment of overall play risk within one software application. Unlike traditional common risk segment maps, the play chance maps are rigorously linked to the data and interpretations used to build them. This provides an audit trail and facilitates efficient updates when new data - or interpretations-become available. 


\section{Introduction}

Common risk segment mapping is typically used to generate a series of $2 \mathrm{D}$ maps that represent the perceived level of risk in elements of the petroleum system, and may also include information pertaining to surface phenomena - such as iceberg flows or mountainous terrain. More often than not these maps are colour coded, with green being low perceived risk, red high risk, and yellow intermediate. In the past these maps were produced on transparencies and overlaid on a light table, to identify the correspondence of green areas and define areas of lower risk. Present industry practice usually involves working in some form of Geographic Information System (GIS) environment, where "traffic light" maps are converted to shape files that are combined electronically to identify areas of perceived low exploration risk, within a play.

Whilst this methodology is widely used, it suffers from some significant disadvantages. Once the shape files are created as "traffic light maps", they become disconnected from the data used to generate them. They also become the end point in a linear workflow - updates with new data require reinitiating the entire workflow process. As such, they are often static snapshots of perceived risk at a fixed point in time. Finally, the superposition of maps of elements of the petroleum system such as source rock, reservoir, and seal presence, overlooks the complexity of petroleum systems where timing and migration are also key elements requiring 4D dynamic analysis, rather than $2 \mathrm{D}$ static representations (Peters et al. 2009).

Explorationists are well aware of these shortcomings, but time and human resources constraints have led to this methodology's frequent use. A recent survey indicates that, worldwide, an average of two out of three wildcat exploration wells fails to find commercial quantities of hydrocarbons, and $75 \%$ of these failures result from incorrect evaluation of charge or seal risk (Kibsgaard, 2011). Clearly, there is a need to improve our assessment of play risk if the industry is to improve its exploration success ratio.

We have developed a system that generates chance of success maps from maps of the physical elements of the petroleum system-including analysis of dynamic charge and seal risk. These maps are expressed in probability units, so that simple map multiplication provides a map of overall play chance of success. Since the maps are derived from data in a software environment that supports model-based integration, the data and interpretations used to generate the play risk assessment are stored and available for audit or revision. Similarly, new data can be readily incorporated and the perceived risk maps of the play updated. Finally, values from these maps can be objectively sampled to provide input to the assessment of various prospects within the play, supporting a rigorous approach to play-based exploration.

\section{Method}

In order to assess all of the elements of the petroleum system (Magoon and Dow, 1994), it is necessary to carry out a dynamic evaluation of petroleum generation, migration, and timing, in addition to evaluating the static physical properties of the source, reservoir, and seal (Peters et al. 2009). Whilst this is widely understood, time and human resource constraints often result in these elements not being considered in the common risk segment approach to assessing play risk. To overcome this constraint we have introduced a "quick look" evaluation of charge and seal risk into our software platform enabling individual petroleum systems to be evaluated using present-day basin geometry. Whilst this is an oversimplification, this approach is sufficient in many basins to answer questions such as:

- Could hydrocarbons have been generated in the basin, and if so when, and where?

- What sort of hydrocarbons?

- Where would they have migrated to?

- Are traps in the play likely to be filled with gas or oil?

- How could the top seal or fault seal's properties have affected trap fill? 
We answer these questions by assigning properties to the source rock and reservoir intervals, as well as assigning sealing properties to the top surface of the reservoir interval. We then use the simulation engine from an established petroleum system modelling application to calculate the volumes and types of hydrocarbon generated in the present-day geometry of the source rock (the burial history of the source rock is not modeled.). The migration and entrapment of the expelled hydrocarbon volumes can then be modelled using simple buoyancy versus capillary entry pressure of the top seal (Figure 1).

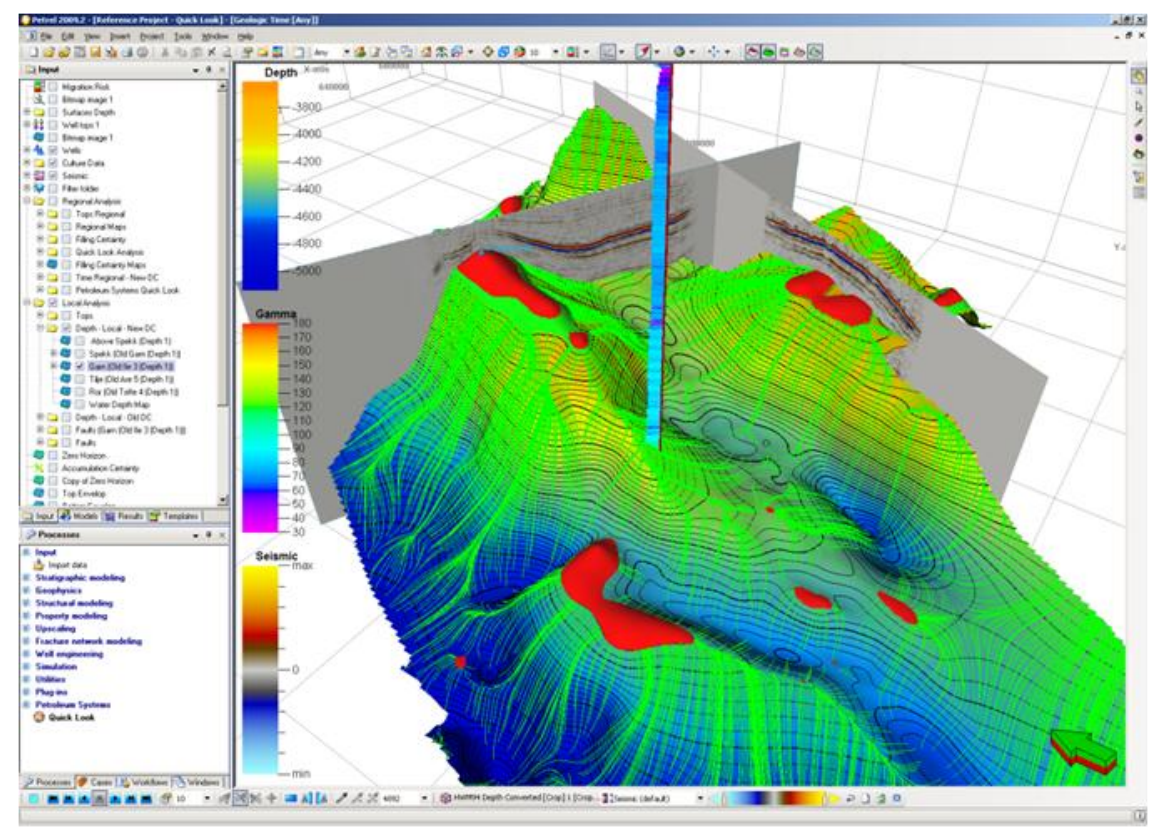

Figure 1. Screen capture illustrating hydrocarbon accumulations (red and green bodies), and migration pathways for hydrocarbon liquids (green lines) generated using the "quick look" approach.

For more complex basins, or where more time is available, more sophisticated modeling of charge and seal may be carried out in a full 3D petroleum system modelling application and brought into our integration platform, via a data exchange plug-in. Timing of generation may be calculated using a simple 1D model, or a full 3D petroleum system simulator to generate an event chart.

In reservoir risk assessment, every play may require slightly different elements to be assessed. For instance, in a fractured carbonate play it will be necessary to evaluate the likely distribution of open natural fractures. Obviously, in a conventional clastic play such maps would not be required to assess play risk. Our system offers flexibility for the user to decide which maps will be combined to provide evaluation of trap, reservoir, charge, seal, and timing or preservation risk (Figure 2).

A simple method is used to transform maps of physical properties to probability maps, as shown in Figure 2. The interpreter assigns a chance of success for high and low values of a mapped property. In this case, reservoir presence is related to a thickness of a sandstone deposited in a shoreface environment. The interpreter has used his experience of the play to assign a chance of success so that a thickness value of $25 \mathrm{~m}$ is assigned a $20 \%$ chance of success (Figure 2). However, once the thickness of the potential reservoir reaches $50 \mathrm{~m}$, the interpreter has assigned a probability of $100 \%$. The rationale behind this might be that where the sandstone is $25 \mathrm{~m}$ thick, or less, then it is not directly mappable from seismic data; but where it exceeds $50 \mathrm{~m}$ in thickness, it is readily mapped. Once these parameters are set, the "Run" button transforms the map of thickness - in meters - to a map displaying the chance that the reservoir is present, in probability units (zero to one) (Figure 3). 


\section{EAGE}

EUROPEAN

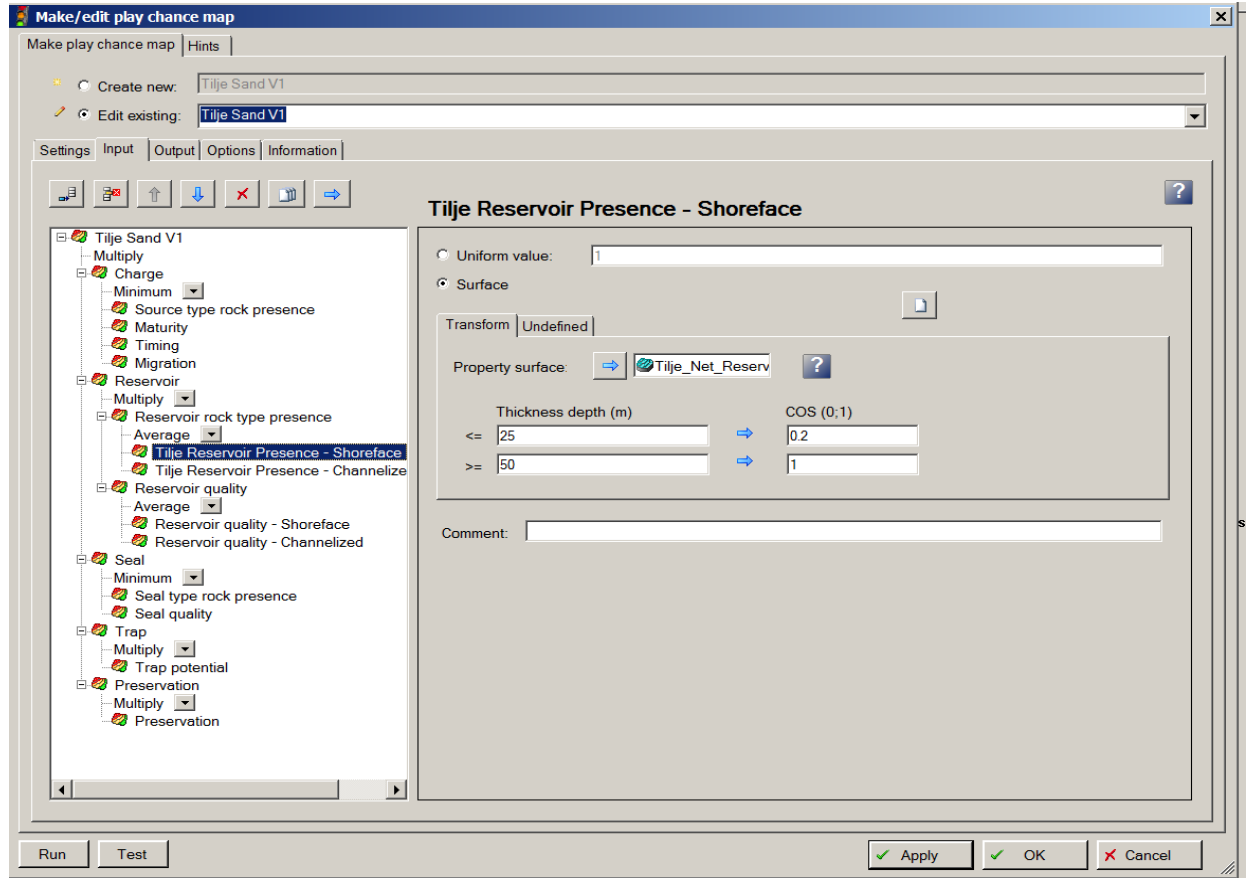

Figure 2. Screen capture illustrating the interface used to generate and combine play chance maps for each of the elements of exploration risk in a play assessment.

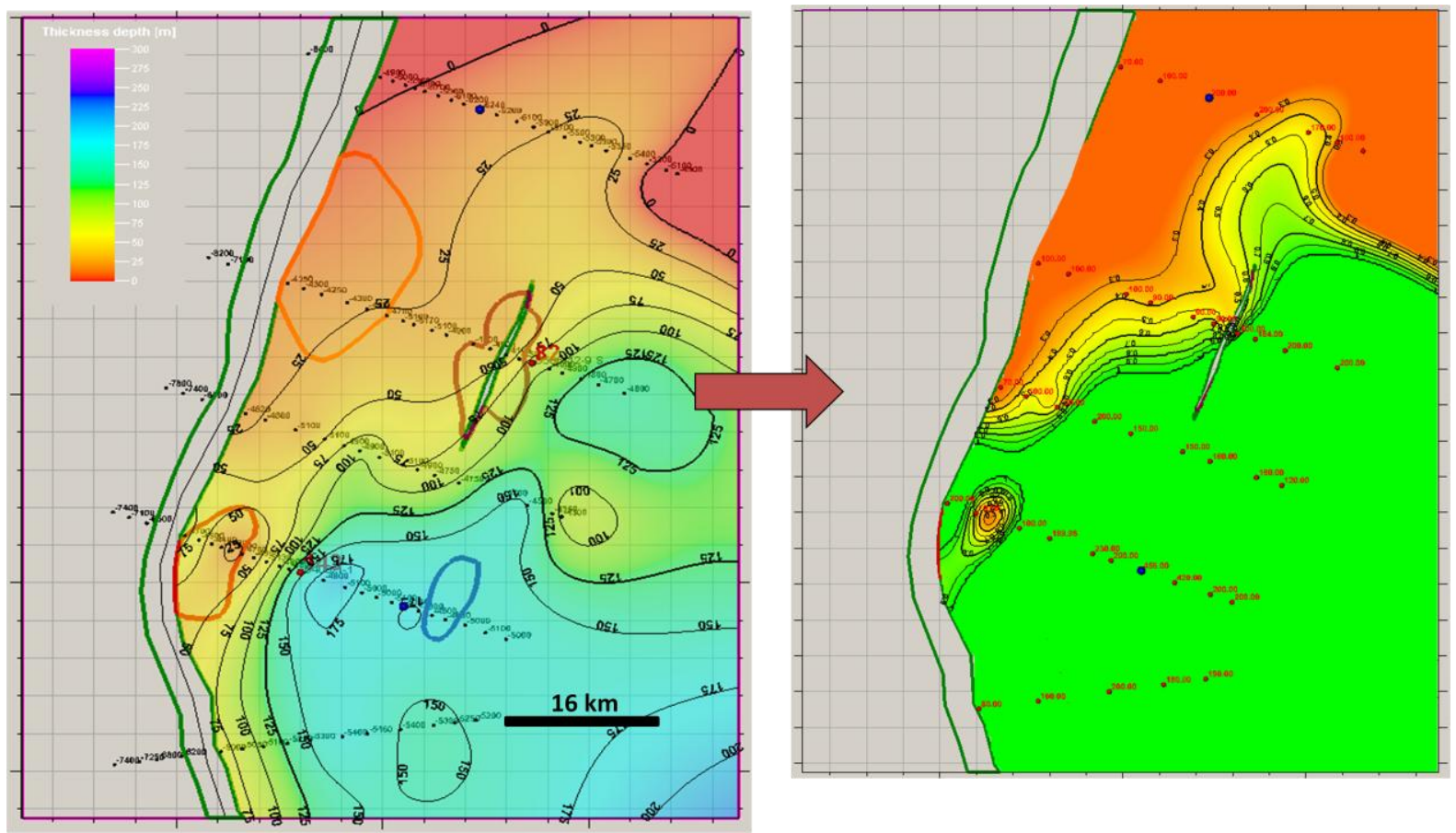

Figure 3. Illustration of how the mapped thickness of the potential reservoir sandstone is converted, using the panel shown in Figure 2, to a chance of success map scaled from zero to one. Note: We have chosen a colour palette that mimics the traditional "traffic light" colours used in common risk segment mapping, but the map actually contains the full information content on thickness variation that was driven by mapping well data. 
Once similar maps have been generated for all elements of the play risk, they are combined using the "Multiply" functionality (Figure 2), to generate a chance of success map for the play.

\section{Conclusions}

We have demonstrated how play chance maps may be generated for every element of subsurface play risk, and combined within one software application to objectively build an assessment of overall play risk. The methodology differs from traditional common segment risk mapping in that the chance of success maps are rigorously connected to the data and interpretation used to generate them. This provides an audit trail and allows efficient updates to the play risk assessment, when new data or interpretations become available. When used in a play-based exploration context these maps may be used to generate input parameters for prospect risk evaluation that are consistent within the play.

\section{References}

Kibsgaard, P., A. [2011] Simmons and Company Energy Conference http://phx.corporateir.net/External.File?item=UGFyZW50SUQ9MTA2MjQ0fENoaWxkSUQ9LTF8VHlwZT0z\&t=1

Peters, K, Schenck, O. and Wygrala, B [2009] Exploration paradigm shift: the dynamic petroleum system. Swiss Bulletin Angew Geology, 14 (1+2) 65-71.

Magoon, L.B. and Dow, W.G. [1994] The petroleum system. In: The petroleum system - from source to trap. AAPG Memoir 60, 3-24. 